\title{
Assessment of von Willebrand Factor/ADAMTS13 Ratio and Vitamin K Levels as Predictor Markers for Severity of COVID-19 Patients
}

\author{
Haneen Saeed Mohsin', Hanaa Addai Ali*, Fadhil Jawad Al-Tu'ma
}

'Department of Chemistry and Biochemistry, College of Medicine, University of Kerbala/Kerbala - Iraq.
2Department of Chemistry, Faculty of Science, University of Kufa/Najaf - Iraq.
${ }^{*}$ Correspondence to: Hanaa Addai Ali (E-mail: muthanahana74@gmail.com)
(Submitted: 20 August 2021 - Revised version received:08 September 2021 - Accepted: 19 September 2021 - Published online: 26 October 2021)

\begin{abstract}
Objectives: To investigate the levels of each of von Willebrand Factor, ADAMTS-13, and vitamin K in sera of Iraqi patients infected with Covid-19 and study the association between them to explore the mechanism roles of micro thrombosis in Covid-19 progression.

Materials and Methods: In this case-control study, a total of (60) COVID-19 patients (min-max) aged (35-65years) who presented within 7-12 days of displaying COVID-19 symptoms were recruited. (30) apparently healthy persons of the same ages and gender were included in this study as a control group. patients were divided into three groups: mild patients group $(n=33)$, sever patients group $(n=15)$, and deceased group $(n=12)$. In this study, the levels of VWF, ADAMTS-13, and vitamin K were measured through enzyme-linked immunosorbent assays (ELISA) in sera from healthy volunteers as a control group, and patients with moderate COVID-19, patients with severe COVID-19, and patients who had deceased from COVID-19. Anthropometric, biochemical data were analyzed.

Results: The levels of VWF, ADAMTS-13, and vitamin K were did differ significantly among groups. However, the level of vWF was significantly higher in moderate COVID-19 and severe cases of COVID-19 groups compared to control indicating it to be an independent predictor in the coronavirus disease. The levels of vitamin $\mathrm{K}$ were significantly lower in the patients group than the control group especially in severe case of the COVID-19 group. In contrast, the level of VWF in deceased COVID-19 patients was significantly higher compared to severe cases. The VWF/ADAMTS13 ratio was higher at presentation in COVID-19 patients who died (26.14 vs $12.34 ; P<.0001)$. Linear regression analysis for VWF levels revealed significant negative correlations with ADAMTS-13, and vit. K, and positive correlations with d-dimer and ferritin levels. However ADAMTS-13 demonstrated significant positive correlations with vit. K levels in patients group.

Conclusion: The correlations of VWF-ADAMTS-13 and vitamin K levels in Covid-19 patients could help better define the pathophysiology, clarify the pathogenesis, improve prediction of clinical prognosis, and better guide thromboprophylaxis and treatment Covid-19 patients, which could be uses as hall mark of severe Covid-19 and provide a rational for combined therapeutic approaches for medical staff. A ratio of VWF/ADAMTS-13 prompt higher in the ratio in thrombolysed patients was associated with increased mortality and morbidity.

Keywords: Covid-19, von Willebrand factor, ADAMTS-13, vitamin K, mortality
\end{abstract}

\section{Introduction}

The severe acute respiratory syndrome-coronavirus type 2 causes Coronavirus Disease 19 (Covid-19), a global pandemic threat (SARS-CoV-2). The spike protein of SARS-CoV-2 interacts to angiotensin-converting enzyme 2 (ACE2) receptors, which play a role in viral entrance. ${ }^{1}$ Covid-19 has a wide range of clinical presentations, from minor symptoms like fever and cough to severe types of pneumonia, which can result in acute respiratory distress, multiorgan failure, and death. ${ }^{2}$ The identification of biomarkers linked with varying disease severity is critical for stratifying patients' risk and devising the most effective medicines, in addition to offering vital insights into the disease mechanisms in play. The characteristics of Covid-disease include severe hypercoagulability and endothelial disturbance, with a high rate of venous thromboembolism routinely recorded, particularly in patients with critical illness requiring intensive care. More recently, pulmonary microvascular thrombosis has been proposed as a cause of illness progression. The lungs were discovered to have indications of thrombotic microangiopathy, although the pulmonary arteries at the hilum of each lung were clear of thromboemboli. CD61 and von Willebrand factor (vWF) immunostaining revealed tiny platelet-rich thrombi inside small arteries of the peripheral parenchyma and alveolar capillaries in all instances. ${ }^{3}$

vWF plays a critical function in initial hemostasis, mediating platelet adhesion and aggregation at vascular injury sites. ${ }^{4}$
VWF is also a sign of endothelium activation, as it is generated in large amounts after vascular injury caused by inflammation. A Disintegrin And Metalloprotease with ThromboSpondin 1 Repeats, Number 13 (ADAMTS-13) regulates the size of vWF multimers, and a severe deficiency of ADAMTS-13 activity below $10 \mathrm{IU} / \mathrm{dL}$ is diagnostic for thrombotic thrombocytopenic purpura (TTP), a severe and life-threatening thrombotic microangiopathy caused by an accumulation of hyperactive ultra-large vWF multi. ${ }^{5}$ In individuals with ischemic stroke and myocardial infarction, low levels of this metalloprotease have been linked to an increased risk of thrombosis. TTP survivors who have lower ADAMTS13 activity during disease remission have a higher risk of TTP-unrelated stroke, while congenital TTP patients who get prophylactic ADAMTS13 replacement therapy had a lower risk of ischemic stroke. Furthermore, as seen in sepsis and overt disseminated intravascular coagulation, an imbalance between high molecular weight VWF multimers and ADAMTS13 may generate a prothrombotic state in inflammatory-induced circumstance. ${ }^{6}$

Vitamin K plays a well-known function in coagulation, which is a delicate balance between clot-promoting and clot-dissolving processes. To perform its principal function, procoagulant factor II (FII; i.e. prothrombin) requires vitamin $\mathrm{K}$-dependent carboxylation. Anticoagulant protein $\mathrm{S}$ requires vitamin $\mathrm{K}$ as a cofactor. In contrast to FII, a major percentage of protein $\mathrm{S}$ is generated outside of the liver in endothelial cells, where it has a local anti-thrombotic effect. Vitamin K 
insufficiency impairs carboxylation of extrahepatic vitamin Kdependent proteins more than hepatic vitamin $\mathrm{K}$-dependent proteins. In a state of low vitaminK, this can contribute to increased thrombogenicity.

The goal of this work was to better understand the pathogenesis of microthrombosis in Covid-19 by focusing on the vWF-ADAMTS13 axis. In order to do so, we measured the VWF multimeric pattern, vWF propeptide, and ADAMTS-13 levels, as well as vitamin $\mathrm{K}$ levels, in individuals with varying degrees of illness severity.

\section{Materials and Methods}

This case control study, a total of 60 patients with COVID-19 with ages ranging from 36-60 years ( 40 males and 20 females) who collected within 7-12 days of showing COVID-19 symptoms were confirmed clinically with COVID-19 according tested positive for SARS-CoV-2 by quantitative RT-PCR and chest $\mathrm{x}$-ray or CT scan. The diagnosis of COVID-19 was done according to the COVID-19 diagnostic criteria reported by $\mathrm{Ma}$ et al. ${ }^{8}$ Patients were recruited from Al-Amal Hospital/ Najaf-Iraq and Al-Hussein teaching hospital, Al-Hussein medical city, Kerbala health directorate/Kerbala - Iraq, within the period from Dec., 2020 and mid-July, 2021 and compared to 60 matched healthy groups as a control. To avoid the influence of other comorbidities, this study excluded subjects with chronic diseases, Alcoholics, liver disease, obese, and smokers. the patients were categorized into subgroups according to Murray score into three groups: Group I (mild group $n=$ 33), Group II (Sevre group $n=15$ ), and group III (deceased group $n=12) .{ }^{9}$ All study procedures were done according to the Helsinki Declaration. Recruited patients and healthy controls gave informed consent for their contribution to this study. This study was done in the Department of chemistry and Biochemistry, College of Medicine, University of Kerbala, Kerbala, Iraq and Department of Laboratory, in Al-Sader Medical City, Najef, Iraq and was approved by the Clinical Research and Ethical Committee Board, College of Medicine, University of Kerbala, Iraq.

Venous Blood samples were collected from patients and control groups. Blood samples were separated in to two tubes $.3 \mathrm{ml}$ allowed to clot for $10-15$ minutes at room temperature before centrifugation for 10 minutes at $(3000 \mathrm{X}$ g) in order provide serum. Then serum samples were separated in to tubes and stored at $-80^{\circ} \mathrm{C}$ until time of analysis. Remaining blood $(2 \mathrm{ml})$ was prepared to measure complete blood count. The concentrations of serum ferritin, and D-dimer levels were measured by fluorescence immunoassay (ichroma ${ }^{\mathrm{TM}}$ ). Levels of vWF, ADAMTS-13, and vitamin K were measured through enzyme-linked immunosorbent assays (ELISA) BT LABChina ELISA Kits. Complete blood count was measured by using auto hematology analyzer (linear, Spain).

\section{Statistical Analysis}

The observed data represented as mean \pm SD were analyzed by using the Statistical Package for the Social Sciences (SPSS). Independent $t$ - test was used to evaluate significant differences between healthy and patients' groups. Pearson correlation coefficient test was applied to mention the statistical relationship (association) between any two variables in present study. The levels of significance of $5 \%(P \leq 0.05)$ and $1 \%(P \leq 0.01)$ were obtained to represent the strength of evidence in support of significant differences between variables.

\section{Results and Discussions}

This study involved 60 patients with verified COVID-19 and 60 healthy participants in total. The average age was 56.896 .32 years, while the median age was 56.485 .41 years. As indicated in table, there were no significant differences in age or gender between COVID-19 patients and healthy volunteers (Table 1).

The data analysis revealed a significantly higher presence of serum D-dimer, ferritin levels, and a significant decrease in vitamin $\mathrm{K}$ levels in COVID-19 patients compared to the control group. The data analysis also revealed a significantly higher presence of serum vWF and a significantly lower level of serum ADAMTS13 in COVID-19 patients compared to the healthy group.

In this investigation, a total of COVID-19 patients were included, with 33 mild, 15 severe, and 12 death cases listed in Table 2. The age and BMI of deceased (death) cases were significantly higher than the other cases, the values of neutrophil, D-dimer, and Ferritin levels in deceased and severe cases were significantly higher than the mild case, categories of COVID-19 patients found low levels of ADAMTS-13 in non-survived and severe cases compared to the mild case, the values of vWF levels in deceased and severe cases were significantly higher than the mild case, and the values of vWF levels.

The biochemical parameters tested were linked to the levels of vWF and ADAMTS-13, as well as vitamin K, in the COVID-19 patients group, as shown in Table 3. Age, BMI, D-dimer, and ferritin levels were all found to have a strong positive connection with vWF levels. In the COVID-19 patients group, however, there were substantial negative relationships between ADAMTS-13 and vitamin K levels.

The best our knowledge, this is the first study in Iraqi patients group infected with COVID-19 that shows a statistically strong significant relationship between serum levels of vWF and ADAMTS-13 in Covid-19 patients.

\begin{tabular}{|c|c|c|c|}
\hline Parameters & $\begin{array}{c}\text { COVID-19 } \\
\text { Patients Group } \\
\text { Mean } \pm \text { SD N = } 60\end{array}$ & $\begin{array}{c}\text { Apparently } \\
\text { Healthy Group } \\
\text { Mean } \pm \text { SD N =60 }\end{array}$ & $P$-value \\
\hline $\begin{array}{l}\text { Age, (years) } \\
\text { Gender, (F/M) }\end{array}$ & $\begin{array}{c}56.89 \pm 6.32 \\
20 / 40\end{array}$ & $\begin{array}{c}56.48 \pm 5.41 \\
20 / 40\end{array}$ & N.S \\
\hline BMI, $\left(\mathrm{kg} / \mathrm{m}^{2}\right)$ & $28.62 \pm 4.31$ & $24.46 \pm 3.11$ & 0.001 \\
\hline D-Dimer, (ng/mL) & $3450.20 \pm 1800.17$ & $269.69 \pm 88.96$ & 0.0001 \\
\hline Ferritin, (ng/mL) & $1081.93 \pm 471.46$ & $106.7 \pm 47.81$ & 0.0001 \\
\hline Vit K, $(p g / m L)$ & $614.32 \pm 106.76$ & $1198.9 \pm 151.59$ & 0.0001 \\
\hline$v W F,(n g / m L)$ & $355.30 \pm 44.45$ & $205.07 \pm 20.98$ & 0.001 \\
\hline ADMTS13, (ng/mL) & $19.92 \pm 9.44$ & $37.10 \pm 7.48$ & 0.0001 \\
\hline vWF/ADAMTS13 & $17.83 \pm 5.63$ & $5.52 \pm 2.96$ & 0.0001 \\
\hline
\end{tabular}

Data represented as Mean \pm SD, SD, Standar deviation, N.: Number of subject. BMI, Body mass index, Vit. K: vitamin K; F: female, M: male, NS: Non-Significant, independent $t$-test, $\mathrm{df}=88$. 


\begin{tabular}{|c|c|c|c|c|}
\hline \multirow{2}{*}{ Parameters } & \multicolumn{3}{|c|}{ COVID-19 patients groups } & \multirow{2}{*}{$P$-value } \\
\hline & Deceased $N=12$ & Severe $N=15$ & Mild $N=33$ & \\
\hline Age, (years) & $61.91 \pm 3.42$ & $60.82 \pm 3.11$ & $46.21 \pm 7.50$ & $\begin{array}{l}a=0.09 \\
b=0.00 \\
c=0.00\end{array}$ \\
\hline $\mathrm{BMI},\left(\mathrm{kg} / \mathrm{m}^{2}\right)$ & $33.19 \pm 3.35$ & $30.07 \pm 2.18$ & $23.71 \pm 5.01$ & $\begin{array}{l}a=0.01 \\
b=0.00 \\
c=0.00\end{array}$ \\
\hline D-Dimer, (ng/mL) & $5602.6 \pm 2348.30$ & $4065.1 \pm 2220.15$ & $683.4 \pm 301.81$ & $\begin{array}{l}a=0.000 \\
b=0.000 \\
c=0.000\end{array}$ \\
\hline Ferritin, (ng/mL) & $1409.71 \pm 700.29$ & $1187.34 \pm 692.50$ & $648.79 \pm 399.94$ & $\begin{array}{l}a=0.000 \\
b=0.000 \\
c=0.000\end{array}$ \\
\hline Vit. K, (pg/mL) & $608.46 \pm 103.32$ & $611.40 \pm 118.13$ & $623.84 \pm 112.79$ & $\begin{array}{c}a=0.4 \\
b=0.05 \\
c=0.04\end{array}$ \\
\hline$v W F,(n g / m L)$ & $392.01 \pm 35.22$ & $354.11 \pm 21.54$ & $320.01 \pm 24$ & $\begin{array}{l}a=0.01 \\
b=0.01 \\
c=0.03\end{array}$ \\
\hline ADAMTS13 (ng/mL) & $15.09 \pm 2.49$ & $17.98 \pm 4.35$ & $23.78 \pm 10.71$ & $\begin{array}{l}a=0.01 \\
b=0.001 \\
c=0.001\end{array}$ \\
\hline vWF/ADMTS13 & $26.14 \pm 7.91$ & $18.43 \pm 7.56$ & $12.34 \pm 4.02$ & $\begin{array}{c}a=0.001 \\
b=0.0001 \\
c=0.01\end{array}$ \\
\hline
\end{tabular}

Data represented as Mean \pm SD, SD: Standar deviation, N.: Number of subject,

BMI: Body mass index, $a=$ Deceased $\times$ Severe, $b=$ Deceased $\times$ Mild, $c=$ Severe $\times$ Mild

Table 3. The correlations between serum vWF, ADAMTS-13,vitamin K levels with clinical parameters in COVID-19 patients group

\begin{tabular}{lcccccc}
\hline \multirow{2}{*}{ Parameters } & \multicolumn{2}{c}{ vWF (ng/mL) } & \multicolumn{2}{c}{ ADAMTS-13 (ng/mL) } & \multicolumn{2}{c}{ Vit.K, (pg/mL) } \\
\cline { 2 - 7 } & $\boldsymbol{r}$ & $\boldsymbol{P}$-value & $\boldsymbol{r}$ & $\boldsymbol{P}$-value & $\boldsymbol{r}$ & $\boldsymbol{P}$-value \\
\hline Age, (years) & 0.379 & 0.05 & -0.391 & 0.02 & -0.341 & 0.064 \\
BMl, (kg/m) & 0.386 & 0.04 & -0.486 & 0.003 & -0.372 & 0.050 \\
D-dimer, (ng/mL) & 0.473 & 0.001 & -0.546 & 0.000 & -0.487 & 0.001 \\
Ferritin, (ng/mL) & 0.466 & 0.001 & -0.452 & 0.001 & -0.467 & 0.001 \\
ADAMTS13, (ng/Ml) & -0.575 & 0.000 & 1 & - & 0.397 & 0.01 \\
VWF, (ng/mL) & 1 & - & -0.575 & 0.000 & -0.367 & 0.041 \\
Vit. K, (mg/mL) & -0.367 & 0.041 & 0.394 & 0.01 & 1 & - \\
VWF/ADAMTS13 & 0.454 & 0.001 & -0.412 & 0.001 & -0.388 & 0.01 \\
\hline
\end{tabular}

BMI: Body mass index; $r=$ Pearson correlation coefficient

The endothelial cell (EC) monolayerlining vascular channels prevents pathological thrombosis in normal blood vessels. The pulmonary microvasculature of COVID-19 showed severe EC apoptosis and loss of EC tight junction integrity, according to data from an autopsy research. ${ }^{10}$

There are various other aspects associated to aging that could be reasons for higher mortality and morbidity in the elderly, including declining immunity. The average number of comorbid conditions grew with age, and senior Covid-19 patients scored considerably higher than young and middle-aged patients on the performance scale. ${ }^{11}$ Chronic subclinical systemic inflammation, often called as inflammation, is another well-known characteristic of aged immunity. Inflammation is a critical pathogenic mechanism in Covid-19; thus, inflammation is thought to have a role in the inferior prognosis in senior COVID-19 patients. $^{12}$

Endothelial cells express levels of vWF and ADAMTS13. Excess vWF is secreted by endothelial cells when the vascular endothelium is dysregulated in an acute hyperinflammatory environment. The activity of ADAMTS13 reduces in direct proportion to the inflammatory response. ${ }^{13}$ According to this, the difference in vWF and ADAMTS13 activity could be due to ADAMTS13 inhibition and/or deficiency. ${ }^{14}$ 
In COVID-19, the interaction between vWF and ADAMT13 Despite their importance in maintaining hemostasis and preventing unwanted thrombosis, vWFADAMTS13 interactions have gotten little attention in the study of Covid-19 pathophysiology, particularly VTE. Importantly, decreased ADAMTS13 activity has been linked to increased inflammation in a variety of systems. ${ }^{15,16}$

Coronavirus disease, also known as COVID-19, is a multifaceted illness caused by infection with the severe acute respiratory syndrome coronavirus 2 (SARS-COV-2). Interstitial pneumonia is the most common symptom, which can lead to poor gas exchange, severe respiratory failure, and mortality. ${ }^{17}$ The pathophysiology is complex, and in critically ill patients, a variety of hyper-inflammatory responses and abnormalities in the coagulation system have been described. Understanding the immune-inflammatory mechanisms behind Covid-19's clinical symptoms will help researchers find possible pharmaceutical targets and stroke. ${ }^{18}$ as well as metabolic syndrome. ${ }^{19}$ Coagulation proteins and platelets are involved in the formation of thrombus. Platelet-mediated thrombogenesis is the most common type of thrombogenesis in arterial circulation. When extracellular vWF attaches to exposed collagen via its A3 domain, while the free vWF A1 domain binds to platelet glycoprotein Ib, platelet thrombogenesis occurs (GPIb). ${ }^{20}$ During the severe phase of COVID-19, the systemic production of pro-inflammatory mediators inhibits the cleavage of high molecular weight vWF or interferes with the proteolytic interaction with ADAMTS 13 , resulting in thrombosis. ${ }^{21}$ Covid-19 patients' D-dimer levels were similarly raised. Only a portion of the reasons for the high D-dimer levels have been elucidated. D-dimers are formed during the breakdown of fibrin and are used as a marker of fibrinolytic activity. In critical patients or patients with sepsis, a link between pro-inflammatory cytokines and markers of coagulation cascade activation, such as D-dimer, has been discovered..$^{22}$ There is additional evidence that in inflammatory conditions, the alveolar haemostatic balance shifts toward a prothrombotic activity majority. ${ }^{23}$ In individuals with severe sepsis, pro-inflammatory cytokines may also have a role in endothelial damage, as well as activating coagulation and inhibiting fibrinolysis. Covid-19's spike protein interacts to the angiotensin-converting enzyme 2 receptor on endothelial cells, causing death and thrombosis. ${ }^{24}$ Apoptosis of endothelial cells also results in inflammatory cell infiltration, which increases the risk of thrombosis. ${ }^{25}$ As a result of the meta-analysis, ferritin levels in COVID-19 patients with thrombotic problems were higher than those in individuals without, implying that patients with thrombosis are in a state of hyper-inflammation. High blood ferritin levels have been linked to hypertension in several investigations.

COVID-19 has a high rate of thromboembolism. Vitamin $\mathrm{K}$ is vital for coagulation and may also play a function in lung disorders. Vitamin $\mathrm{K}$ is an important component in preventing blood clotting and is a crucial role in the coagulation system, with a link recently discovered between vitamin $\mathrm{K}$ shortage and the worst COVID-19 results. ${ }^{7}$

The respiratory symptoms of COVID-19 are varied and can sometimes lead to significant consequences. Severe forms of Covid-19, like other severe respiratory disorders, include pneumonia, acute lung injury (ALI), ARDS, and sepsis, which can lead to multiple organ failure and death. ${ }^{2}$ According to studies, the respiratory symptoms might deteriorate as quickly as 9 days after the onset of ARDS. ${ }^{26}$ Even in asymptomatic patients, computed tomography (CT) scans revealed damage to the lungs, defined by pulmonary ground glass opacification, demonstrating that the multitude of Covid-19-related problems is still far from being fully recognized.

\section{Conclusion}

In conclusion presentation a strong association between the levels of VWF, ADAMTS13, and vitamin K on admission with respect to the severity, functional outcome, mortality and better guide thromboprophylaxis and consequential clinical recovery and may be used for future prognostication and guidance of therapy. A ratio of $\mathrm{vWF} / \mathrm{ADAMTS}-13$ was associated with increased mortality and morbidity. However, additional investigations are needed to be performed to understand the exact cellular mechanism of this disease.

\section{Acknowledgments}

All authors would like to thank the participated patients and the team of COVID-19 Centers for their support during this study.

\section{Conflicts of Interest}

No conflicts of interest regarding the publication of this article.

\section{References}

1. Guan, W.-j., Ni, Z.-y., Hu, Y., et al. (2020). Clinical characteristics of coronavirus disease 2019 in China. New England Journal of Medicine, 382(18), 1708-1720.

2. Zaim, S., Chong, J. H., Sankaranarayanan, V., et al. (2020). COVID-19 and multiorgan response. Current Problems in Cardiology, 45(8), 100618.

3. Fox, S. E., Akmatbekov, A., Harbert, J. L., et al. (2020). Pulmonary and cardiac pathology in African American patients with COVID-19: an autopsy series from New Orleans. The Lancet Respiratory Medicine, 8(7), 681-686.

4. Ruggeri, Z. M., (2003). Von Willebrand factor, platelets and endothelial cell interactions. Journal of Thrombosis and Haemostasis. 1(7), 1335-1342.

5. Scully, M., Cataland, S., Coppo, P., et al. (2017). Consensus on the standardization of terminology in thrombotic thrombocytopenic purpura and related thrombotic microangiopathies. Journal of Thrombosis and Haemostasis, 15(2), 312-322.
6. Singh, K., Kwong, A. C., Madarati, H., et al. (2021). Characterization of ADAMTS13 and von Willebrand factor levels in septic and non-septic ICU patients. Plos One, 16(2), e0247017.

7. Dofferhoff, A. S., Piscaer, I., Schurgers, L. J., et al. (2020). Reduced vitamin K status as a potentially modifiable risk factor of severe COVID-19. Clinical Infectious Diseases: an Official Publication of the Infectious Diseases Society of America.

8. Ma, L.-L., Li, B.-H., Jin, Y.-H., et al. (2020). Developments, evolution, and implications of national diagnostic criteria for COVID-19 in china. Frontiers in Medicine, 7, 242.

9. Murray, J. F., Matthay, M. A., Luce, J. M., et al. (1988). An expanded definition of the adult respiratory distress syndrome. Am Rev Respir Dis, 138(3), $720-723$. 
10. Wichmann, D., Sperhake, J.-P., Lütgehetmann, M., et al. (2020). Autopsy findings and venous thromboembolism in patients with COVID-19: a prospective cohort study. Annals of Internal Medicine, 173(4), 268-277.

11. Kim, D., Lee J. Y., Yang J. S., et al. (2020). The architecture of SARS-CoV-2 transcriptome. Cell, 181(4), 914-921.

12. Bonafè, M., Prattichizzo, F., Giuliani, A., et al. (2020). Inflamm-aging: why older men are the most susceptible to SARS-CoV-2 complicated outcomes. Cytokine \& Growth Factor Reviews, 53, 33-37.

13. Katneni, U. K., Alexaki, A, Hunt, R. C., et al. (2020). Coagulopathy and thrombosis as a result of severe COVID-19 infection: a microvascular focus. Thrombosis and Haemostasis.

14. Marco, A., \& Marco, P. (2021). Von Willebrand factor and ADAMTS13 activity as clinical severity markers in patients with COVID-19. Journal of Thrombosis and Thrombolysis, 1-7.

15. Liu, C., Zhao, L., Zhao, J., et al. (2017). Reduced ADAMTS-13 level negatively correlates with inflammation factors in plasma of acute myeloid leukemia patients. Leukemia Research, 53, 57-64

16. Takaya, H., Kawaratani, H., Kubo, T., et al. (2018). Platelet hyperaggregability is associated with decreased ADAMTS13 activity and enhanced endotoxemia in patients with acute cholangitis. Hepatology Research, 48(3), E52-E60.

17. Helms, J., Kremer, S., Merdji, H., et al. (2020). Neurologic features in severe SARS-CoV-2 infection. New England Journal of Medicine, 382(23), 2268-2270.

18. Vergouwen, M. D., Meijers, J. C., Geskus, R. B., et al. (2009). Biologic effects of simvastatin in patients with aneurysmal subarachnoid hemorrhage: a double-blind, placebo-controlled randomized trial. Journal of Cerebral Blood Flow \& Metabolism, 29(8), 1444-1453.

19. Ziliotto, N., Bernardi, F., Jakimovski, D., et al. (2018). Hemostasis biomarkers in multiple sclerosis. European Journal of Neurology, 25(9), 1169-1176.

20. Zhu, S., Gilbert, J. C., Hatala, P., et al. (2020). The development and characterization of a long acting anti-thrombotic von Willebrand factor (VWF) aptamer. Journal of Thrombosis and Haemostasis, 18(5), 1113-1123.

21. Neerukonda, S.N., Katneni, U. (2020). A review on SARS-CoV-2 virology, pathophysiology, animal models, and anti-viral interventions. Pathogens, 9(6), 426.

22. Borjini, N., Fernández, M., Giardino, L., et al. (2016). Cytokine and chemokine alterations in tissue, CSF, and plasma in early presymptomatic phase of experimental allergic encephalomyelitis (EAE), in a rat model of multiple sclerosis. Journal of Neuroinflammation, 13(1), 1-16.

23. Omata, M., Cheng, A. L., Kokudo, N., et al. (2017). Asia-Pacific clinical practice guidelines on the management of hepatocellular carcinoma: a 2017 update. Hepatology International, 11(4), 317-370.

24. Ferrario, C. M., Jessup, J., Chappell M. C., et al. (2005). Effect of angiotensinconverting enzyme inhibition and angiotensin $\|$ receptor blockers on cardiac angiotensin-converting enzyme 2. Circulation, 111(20), 2605-2610.

25. Connors, J. M., \& Levy, J. H. (2020). Thromboinflammation and the hypercoagulability of COVID-19. J Thromb Haemost, 18(7), 1559-1561.

26. Wu, A., Peng, Y., Huang, B., et al. (2020). Genome composition and divergence of the novel coronavirus (2019-nCoV) originating in China. Cell Host \& Microbe. 27(3), 325-328. 